\title{
Oxygen use in acute myocardial infarction - an online survey of health professionals' practice and beliefs.
}

\author{
Amanda Burls, José I. Emparanza, Tom Quinn, Juan B. Cabello
}

Centre for Evidence-Based Medicine, Department of Primary Health Care, University of Oxford, Old Road Campus, Oxford OX3 7LF, UK

Amanda Burls

Director of Postgraduate Programmes in Evidence-Based Health Care

Unidad de Epidemiología Clínica e Investigación, CASPe, CIBER-ESP, Hospital Donostia, PO Beguiristain S/N, 20014 San Sebastián, Spain

José I. Emparanza

Clinical epidemiologist and consultant paediatrician

Division of Health and Social Care, Faculty of Health and Medical Sciences, University of Surrey, Guildford, GU2 7XH, UK

Tom Quinn

Professor of clinical practice

Centro coordinador CASPe \& Cardiology Department

Hospital General Universitario de Alicante. Calle Maestro Alonso, 030010 Alicante, Spain

Juan B. Cabello

Clinical epidemiologist and consultant cardiologist

Corresponding author: Professor Tom Quinn

Email: t.quinn@surrey.ac.uk Tel: 01483684553

Dr Burls is the guarantor of this paper.

Key words: acute myocardial infarction, oxygen inhalation therapy, uncertainty.

Word count 2,437 
Abstract

Introduction

There is growing interest in the safety of oxygen therapy in emergency patients. $A$

Cochrane review of oxygen versus air for patients with acute myocardial infarction (AMI) showed a potentially important, but statistically non-significant, increase in mortality (RR $3.03(95 \% \mathrm{Cl}$ 0.93-9.83)) and concluded a definitive randomised controlled trial (RCT) was needed.

\section{Objective}

To explore the feasibility of conducting an RCT of oxygen versus air in AMI, by exploring the beliefs of UK professionals who treat patients with AMI about oxygen's benefits, and to establish a baseline of reported practice by asking about their use of oxygen.

\section{Method}

A cross-sectional online survey of UK emergency department, cardiology and ambulance staff.

\section{Result}

524 responses were received. All specialities had over 100 respondents. $98.3 \%$ said they always or usually use oxygen. $80 \%$ reported having local guidelines that recommended the routine use of oxygen. $55 \%$ believed oxygen definitely or probably significantly reduces the risk of death while only $1.3 \%$ reported that they thought "it may even increase the risk of death". There were only minor differences across specialties and grades.

\section{Conclusion}

Widespread belief in the benefit of oxygen in AMI, may make it difficult to persuade funders of the importance of this issue and health professionals to participate in enrolling patients into a trial in which oxygen would be withheld from half their patients. (209 words) 
Introduction

In 2007 articles suggesting that oxygen may harm people who have had a heart attack appeared in the UK popular press following the publication of a narrative review of oxygen use in acute myocardial infarction (AMI).[1]

National and international guidelines have until recently recommended use of oxygen in AMI patients in both the pre-hospital and hospital setting. [2-5] We undertook a Cochrane systematic review of randomised control trials (RCTs) on this topic.[6] The review concluded that there was considerable uncertainty with a relative risk (RR) of death for oxygen compared to air of $3.03(95 \% \mathrm{Cl} 0.93-9.83)$, and that, since it was possible that oxygen increases mortality, a definitive RCT was required. [6]

Objectives

- To assess the feasibility of an RCT by examining potential professional resistance

- To see how frequently oxygen therapy is reported as being used in patients with AMI

- To explore health professionals' beliefs about the benefits or harms of oxygen in terms of death and pain relief and their relationship to practice

- To establish a baseline to later explore variations in reported practice and beliefs between countries and whether publication of the Cochrane review altered perceptions.

Methods

Design

A cross-sectional online survey of UK professionals who treat patients with AMI.

Population surveyed

We used theoretical sampling. The most important professional groups in the UK treating AMI were identified as ambulance, emergency department (ED), and cardiology staff. Professional groups were accessed by:

- Asking the British Cardiovascular Society (BCS) to place a link to the survey on their website. This appeared on the homepage as the first item on the central "what's new" section. Members were alerted to the survey and encouraged to take part via the BCS newsletter.

- The Joint Royal Colleges Ambulance Liaison Committee (JRCALC), the British Paramedic Association, the British Association for Nursing in Cardiovascular Care and the Royal College of Nursing's Cardiovascular Network disseminated the survey to their members using email.

- A notice and link were placed on the National Library for Health Cardiovascular Specialist Library webpage encouraging people to take the survey.

- Regional mailing lists of cardiology contacts were used.

- Some health professionals were alerted to the survey by colleagues.

The survey was launched at the beginning of April 2007 and the above actions taken during the following month. The survey closed on 10th May 2007. 
Survey characteristics

To maximise completion rates we restricted the survey to seven questions. Questions 1, $5,6,7$ had a free text option in addition to specified categories. No question required a mandatory response.

People were prevented from completing the survey more than once using cookies. People who left the survey half-way through were able to return to the question at which they stopped and complete the survey.

We used Surveyselect.net ${ }^{\circledR}$ software hosted on a university server.

\section{Ethical approval}

To ensure the survey met best practice [7-10], a draft was sent to colleagues for comment prior to launch. It was confirmed that ethical committee approval was not required for this study in the UK. Participants were invited to leave their email addresses to receive the results of the survey.

\section{Analysis}

Data were analysed using Systat ${ }^{\circledR}$ according to a pre-specified strategy. Email addresses were stripped prior to analysis to maintain confidentiality.

Answers were described in absolute numbers and percentages. Chi-squared was used to compare frequencies between groups. Where numbers were insufficient, meaningful categories were aggregated and/or Fischer's Exact test was used. For hypothesis generating analyses we aggregated the behavioural and belief data into binary variables: those who said that they "always", "always unless contraindicated" or "usually" routinely gave oxygen to patients with AMI were designated as "use oxygen"; those who "usually" or "almost always" thought that giving oxygen reduces pain were designated "improves pain"; those who thought it "definitely" or "probably significantly reduces the risk of death" were designated as "reduces death". P-values are reported when less than 0.20 otherwise the result is reported as "not significant".

Results

The survey was online for 40 days. There were approximately 48 hours of downtime during this period while the server was being physically relocated. At close there were 526 records in the database. Two contained nonsense not logically possible from the survey structure. We attributed these to technical problems (possibly caused by the relocation of the server) and deleted them. There were therefore 524 valid responses. Given the recruitment methods, it was not possible to report the response rate of those aware of and eligible to complete the survey. The question response rate dropped as the survey progressed with $75.5 \%$ answering the last question. Questions left blank were reclassified to "don't know" for questions 3-5. There was a good response from all three professional groups: 104 responses from ED; 153 from cardiology; 151 from the ambulance service. 198 people left their email address.

All questions and responses are given in table $\mathbf{1 .}$ 


\section{Overall result}

Of those who see patients with AMI, 98.3\% said they usually or always use oxygen. 55\% of all respondents believed that oxygen definitely or probably significantly reduces the risk of death, contrasted with $1.3 \%$ (7 people) who believed it may increase death. Of the latter, three usually or always administered oxygen. $29.4 \%$ believed it always or usually reduces pain. No-one thought it increases pain.

Only $1.7 \%$ said there were no local guidelines and $96 \%$ of local guidelines were said to recommend the use of oxygen.

\section{Profession and specialty}

Non-doctors were more likely to believe oxygen reduces death $(69 \%)$ than doctors $(50 \%)$ $(\mathrm{P}=0.02)$. Moreover $8.3 \%$ of doctors thought that oxygen might actually increase mortality compare to just $0.98 \%$ of non-doctors $(P<0.01)$. There was no statistically significant difference, however, between professions in their behaviour or their beliefs about the effect of oxygen on pain (Table 2).

ED and ambulance staff reported similar behaviours and beliefs. Cardiology staff were, however, marginally less likely to use oxygen than others (96\% vs. $100 \% \mathrm{P}<0.003)$ and considerably more sceptical about whether it reduces mortality ( $48 \%$ vs. $74 \% \mathrm{p}<0.01)$

\section{(Table 2).}

Table 1 - Survey answers

\begin{tabular}{|c|c|c|c|}
\hline Question & Possible answers & | $\mathrm{N}$ & $\%$ \\
\hline 1. In your clinical & Never & $\mid 1$ & 0.2 \\
\hline & $\begin{array}{l}\text { Only if there is an additional } \\
\text { indication }\end{array}$ & 4 & 0.8 \\
\hline & Occasionally & 4 & 0.8 \\
\hline & Usually & $\mid 30$ & 5.7 \\
\hline & Always unless contraindicated & $\mid 159$ & 30.3 \\
\hline & Always & $\mid 319$ & 60.9 \\
\hline & Not applicable & $\mid 7$ & 1.3 \\
\hline 2. How many patients & 0 & 15 & 2.9 \\
\hline & $1-10$ & 98 & 18.7 \\
\hline & $11-25$ & | 94 & 17.9 \\
\hline & $26-50$ & $\mid 92$ & 17.6 \\
\hline & More than 50 & $\mid 177$ & 33.8 \\
\hline & Left blank & 48 & 9.2 \\
\hline 3. Do you believe & Yes, almost always & 41 & 7.8 \\
\hline & Usually & 113 & 21.6 \\
\hline & Sometimes & | 219 & 41.8 \\
\hline & Rarely & 52 & 9.9 \\
\hline
\end{tabular}




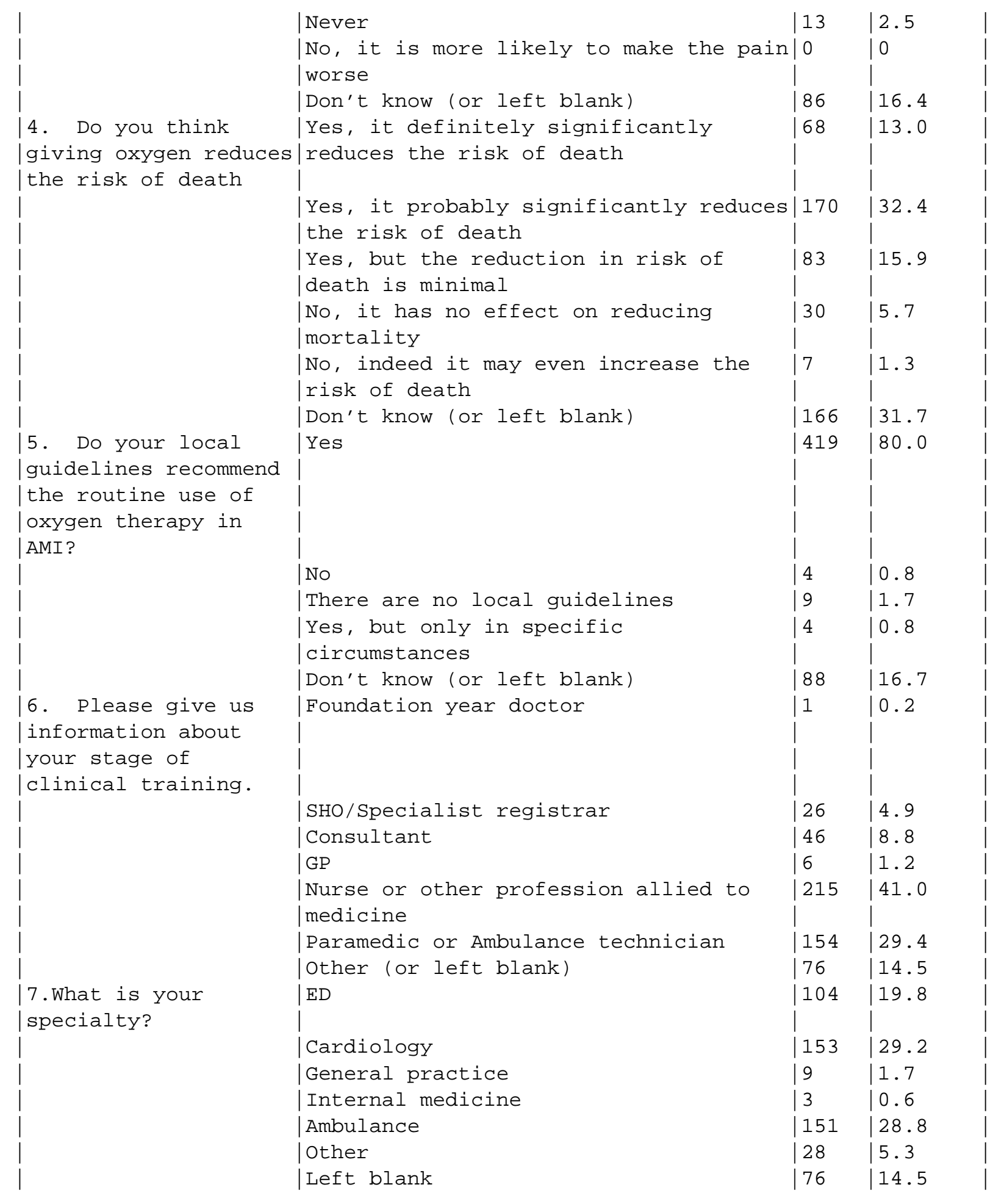

Table 2 - Comparisons of differences in behaviour and beliefs between specialties and profession

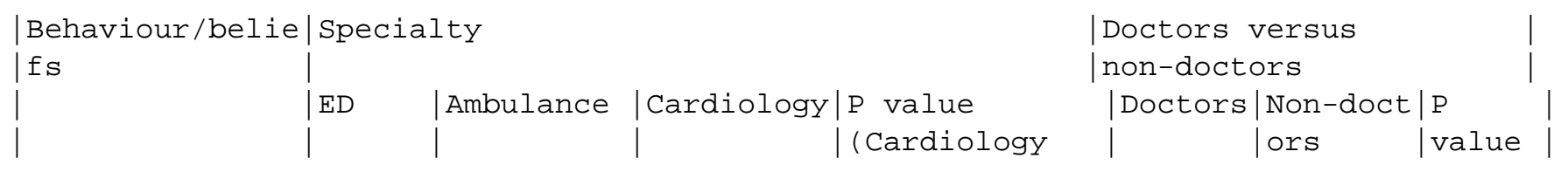




\begin{tabular}{|c|c|c|c|c|c|c|c|}
\hline & & & & (vs. others) & & & \\
\hline Use oxygen & $100 \%$ & $100 \%$ & $96.0 \%$ & $P<0.003$ & $95.8 \%$ & $99.2 \%$ & 0.06 \\
\hline Improves pain & $37.9 \%$ & $34.7 \%$ & $35.9 \%$ & ns & $25.4 \%$ & $37.0 \%$ & 0.11 \\
\hline Reduces death & $74.4 \%$ & $74.1 \%$ & $48.0 \%$ & $\mid P<0.01$ & $50.0 \%$ & $69.0 \%$ & 0.02 \\
\hline
\end{tabular}

All of the four who reported that they did not routinely use oxygen or believe it reduced pain or death were from cardiology: two consultants; one nurse; one senior house officer.

Experience, seniority and volume

Our prior hypothesis was that senior staff, and those who treated large numbers of patients, would be would be more sceptical about the benefits and use of oxygen, as they would tend to be more familiar with the lack of evidence to support the practice. Those seeing more than 50 patients a year reported a small, but statistically significantly, lower use of oxygen. This group was also less likely to believe that it improved pain and reduced death although the differences were not significant (Table 3).

Doctors who had finished specialist training were less likely to believe that oxygen improved pain but there was no significant difference in behaviour or beliefs about the effect on mortality (Table 3).

Table 3 - Comparisons of differences in beliefs and behaviour by experience

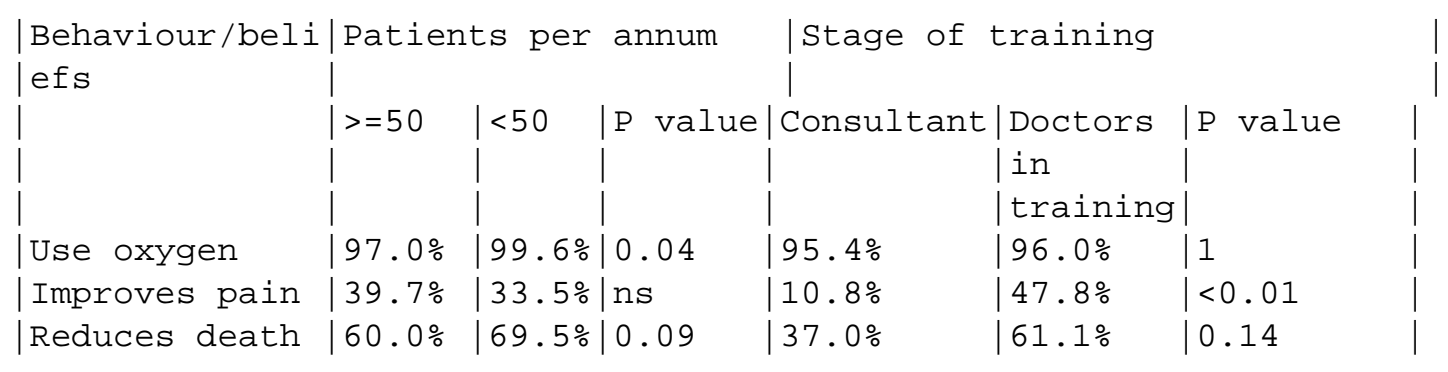

Ambulance staff, whether paramedics or technicians, reported $100 \%$ use of oxygen. Paramedics were more sceptical about oxygen's benefits than technicians although the difference was not statistically significant (Table 4).

Table 4 - Comparison of behaviour and beliefs of ambulance staff

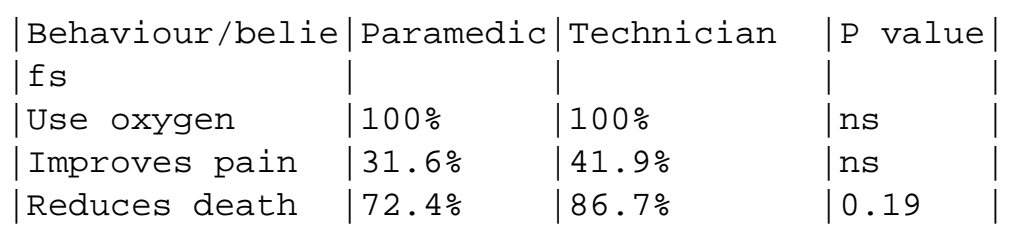

Discussion

Our survey demonstrated that the majority of respondents believe oxygen to be beneficial and nearly all report using it routinely, even those who do not believe in its benefits. This is consistent with the recommendations for the management of AMI in 
most current guidelines at the time we undertook the survey. The overwhelming majority of respondents said there were local guidelines of which only four were reported not to recommend the use of oxygen. Although the results were highly statistically significant $(P<0.003)$, there was negligible difference between specialities with $100 \%$ of ED and ambulance staff usually or almost always using oxygen compared to $96 \%$ of cardiology staff.

The use of the internet for health research is growing and unstoppable. The speed, ease of conduct and cost-effectiveness of online surveys make them attractive. A strength of this survey is that there was good response from across all key professional groups. However there are known methodological challenges with online open-access surveys, [7] and this study suffers in particular from two limitations: we could not ensure that those responding actually came from the professional groups they claim to come from; the sampling is not random. To limit the former problem we used targeted methods to raise awareness of the study. The consistency and coherence of results suggests to us that it is unlikely that there is a significant proportion of false respondents. (Also 200 people left email addresses, many of which were NHS.) The second problem, that random sampling, as commonly used in traditional surveys, is not possible when using the internet signposting, webpage advertising, or e-mail snowballing techniques, for recruiting participants, means that there is not a numerically identifiable, probabilistically selected, denominator population. This limits the statistical inferences that can be appropriately drawn. We used theoretical sampling to select key professional groups to be surveyed, choosing those most likely to see patients with AMI, in order to capture, not only the use of oxygen, but, more importantly, subjective beliefs and rationales behind such use. While it could be speculated that professionals with strong opinions would be more likely to respond to this survey, we do not think there is a compelling a priori reason to suggest that online survey methods will be more likely to systematically select a professional group more likely to report the observed results (a belief in the benefits, and reported use, of oxygen) than traditional survey methods.

There were too few people who were sufficiently sceptical about the value of oxygen to permit deep exploration.

In this study $98 \%$ of respondents reported usually or always using oxygen compare to the 1997 UK survey which reported that oxygen was not routinely prescribed in $53 \%$ of UK coronary care units. [11] The difference may be due to today's greater emphasis on following guidelines compared to a decade ago but it could also be a reporting bias because people wish to appear compliant with recommended practice.

The results of this study implied that there could be significant resistance from professionals to an RCT looking at the benefit of oxygen because they may have insufficient equipoise to be prepared to withhold oxygen from the control group. Most major guidelines current when we undertook the survey would reinforce this. The American College of Cardiology identified oxygen as a 'routine measure', with a Class I recommendation that it should be administered to patients with arterial oxygen desaturation ( $\mathrm{SaO} 2$ less than $90 \%$ ) based on [level of evidence $\mathrm{B}$ ], and a lla 
recommendation that it is "reasonable' to administer to all uncomplicated STEMI patients during the first 6 hours" based on [level of evidence C]. [2,3] The European Society of Cardiology recommended use at 2-4 litres/min "especially to those who are breathless or who have any features of heart failure and shock" and comment on the value of monitoring saturation to help in deciding on the need for oxygen administration. [4] The International Liaison Committee on Resuscitation (ILCOR) presented a "consensus on science" in which they reflect on one animal study suggesting reduced infarct size and one human study reporting improvements in the ECG, and a single randomised trial which failed to show long term benefit; the subsequent recommendation is that oxygen should be given to desaturated patients but goes on to comment "Given the safety profile of oxygen in the (MI) population and potential benefit in patients with unrecognised hypoxia, it is reasonable to give to all uncomplicated STEMI patients during the first 6 hours of emergency management." [5] The European Resuscitation Council suggested 4$8 \mathrm{l} / \mathrm{min}$ for all desaturated patients and those with pulmonary congestion, and, while explicitly acknowledging the 'lack of proof' for long-term benefit in uncomplicated patients, recommend it be given to all on the basis that it will benefit those with unrecognised hypoxia. [12] JRCALC, which publishes UK national guidelines for treatments delivered by paramedics, recommended routine use of high concentration oxygen, whatever the oxygen saturation, if an acute coronary syndrome (ACS) is suspected. [13]

That an overwhelming majority of participants in our survey reported routine use of oxygen can be viewed positively in that compliance with guidelines is high. However, the above international recommendations appear to reflect consensus rather than high quality evidence. Our findings suggest that some professionals follow guidelines despite their personal beliefs about the intervention's value. There is a need, therefore, to critically assess the guidelines on which such treatment is based in an area where concerns about safety and efficacy have been resurrected some decades since the best $\mathrm{RCT}$ reported absence of benefit.

This is beginning to happen. With the growing emphasis on evidence-based practice, new guidelines are beginning to diverge from the consensus. Recent guidelines from the Scottish Intercollegiate Guidelines Network (SIGN) unequivocally state that there is no evidence to support routine use of oxygen across the broad spectrum of ACS, restricting recommendations for use in hypoxic patients and those with pulmonary oedema or continuing myocardial ischaemia, and making clear that this recommendation is based on expert opinion alone. [14] Similarly the recent European guideline does not recommend routine oxygen use in uncomplicated ACS. [15] The British Thoracic Society have recently published guidelines on emergency oxygen use in adult patients, endorsed by several organisations including JRCALC, recommending that MI or ACS patients should only receive oxygen if hypoxaemic, although this again is based on expert consensus rather than hard evidence. [16]

The diversity of approaches to oxygen use, in relation to indications, dosage and duration of treatment, in the major guidelines imply that not all can be recommending what is best for patients. Moreover there is the potential for confusion among those providing 
emergency cardiac care. There is a need to adopt a more consistent approach, but this is difficult in the absence of clear evidence of which patients benefit, the appropriate dose, and duration of treatment. RCTs are urgently required to clarify the role of this key component of emergency care.

\section{Conclusion}

The conflict between the current, albeit weak, evidence suggestive of possible harm from oxygen use in $\mathrm{AMI}$, and most current guidelines and practice promoting its use, means that a definitive RCT is urgently required. The widespread belief in the benefit of oxygen, as demonstrated by this survey, suggests that currently it may be difficult to persuade funders of the importance of this issue or health professionals to participate in enrolling patients into such a trial. However the fact that new guidelines are beginning to change their recommendations, despite no new evidence having emerged, suggests that attitudes may be changing. An active educational and publicity campaign to make professionals aware of the current evidence may help spread an appreciation of the uncertainties in this area and make it feasible to conduct the long-needed definitive RCT on this question.

\section{Declaration of competing interests:}

The authors have no competing financial interests in this topic. They have participated in the preparation of a protocol for a randomised controlled trial to investigate the benefits of routine administration of inhaled normotensive oxygen in uncomplicated acute myocardial infarction. TQ is involved in developing JRCALC guidelines and helped to write the CHD NSF.

\section{Funding}

None. 


\section{References}

1. Beasley $R$, Aldington $S$, Weatherall $M$ et al Oxygen therapy in myocardial infarction: an historical perspective. J R Soc Med 2007;100:130-33

2. Antman EM, Anbe DT, Armstrong PW et al ACC/AHA guidelines for the management of patients with ST-elevation myocardial infarction--executive summary: a report of the American College of Cardiology/American Heart Association Task Force on Practice Guidelines (Writing Committee to Revise the 1999 Guidelines for the Management of Patients With Acute Myocardial Infarction) Circulation 2004;110:588-636.

3. Anderson JL, Adams CD, Antman EM et al ACC/AHA 2007 guidelines for the management of patients with unstable angina/non-ST-Elevation myocardial infarction: a report of the American College of Cardiology/American Heart Association Task Force on Practice Guidelines (Writing Committee to Revise the 2002 Guidelines for the Management of Patients With Unstable Angina/Non-STElevation Myocardial Infarction) developed in collaboration with the American College of Emergency Physicians, the Society for Cardiovascular Angiography and Interventions, and the Society of Thoracic Surgeons endorsed by the American Association of Cardiovascular and Pulmonary Rehabilitation and the Society for Academic Emergency Medicine. J Am Coll Cardiol 2007; 50:e1-e157.

4. Van de Werf F, Ardissino D, Betriu A et al Management of acute myocardial infarction in patients presenting with ST-segment elevation. The Task Force on the Management of Acute Myocardial Infarction of the European Society of Cardiology. Eur Heart J 2003;24:28-66.

5. International Liaison Committee on Resuscitation. 2005 International Consensus on Cardiopulmonary Resuscitation and Emergency Cardiovascular Care Science with Treatment Recommendations. Part 5: acute coronary syndromes. Resuscitation 2005; 67:249-269.

6. Burls A, Bayliss S, Emparanza JI, Quinn T. Oxygen therapy for acute myocardial infarction (Protocol). Cochrane Database of Systematic Reviews 2008, Issue 2. Art. No.: CD007160. DOI: 10.1002/14651858.CD007160.

7. Eysenbach G. Improving the quality of Web surveys: the Checklist for Reporting Results of Internet E-Surveys (CHERRIES) J Med Internet Res 2004;6:e34

8. Schonlau M. Will web surveys ever become part of mainstream research? J Med Internet Res 2004;6:e31.

9. Schleyer TK, Forrest JL. Methods for the design and administration of web-based surveys 2. J Am Med Inform Assoc 2000;7:416-425.

10. Ekman A, Klint A, Dickman PW et al Optimizing the design of web-based 
questionnaires--experience from a population-based study among 50,000 women 2. Eur J Epidemiol 2007;22:293-300

11. Wilson AT, Channer KS. Hypoxaemia and supplemental oxygen therapy in the first 24 hours after myocardial infarction: the role of pulse oximetry. $J R$ Coll Physicians Lond 1997;31:657-661.

12. Arntz H R, Bossaert L, Filippatos G S. European Resuscitation Council Guidelines for Resuscitation 2005 Section 5. Initial management of acute coronary syndromes. Resuscitation 2005;67:S1:S87-S96

13. Scottish Intercollegiate Guidelines Network. Acute coronary syndrome. A national clinical guideline. 2007.

14. Joint Royal Colleges Ambulance Liaison Committee. UK ambulance service clinical practice guidelines 2006.

http://www2.warwick.ac.uk/fac/med/research/hsri/emergencycare/prehospitalcare/jr calcstakeholderwebsite/guidelines/acute_coronary_syndrome_2006.pdf (accessed 21st January 2009)

15. Bassand JP, Hamm CW, Ardissino D et al Guidelines for the diagnosis and treatment of non-ST-segment elevation acute coronary syndromes. Eur Heart J 2007;28:1598-1660

16. O'Driscoll BR, Howard LS, Davison AG on behalf of the British Thoracic Society. BTS guideline for emergency oxygen use in adult patients. Thorax 2008;63 (Suppl VI):Vi1-Vi68

"[The corresponding author has the right to grant on behalf of all authors and does grant on behalf of all authors, an exclusive licence (or non exclusive for government employees) on a worldwide basis to the BMJ Publishing Group Ltd to permit this article (if accepted) to be published in BMJ editions and any other BMJPGL products and sublicences such use and exploit all subsidiary rights, as set out in our licence http://resources.bmj.com/bmj/authors/checklists-forms/licence-for-publication]." 